\title{
Evaluación del rendimiento seco de la Brachiaria humidicola asociada con plantas leguminosas nativas en Llanos de Coclé, Panamá
}

\author{
Evaluation of the dry yield of Brachiaria humidicola associated with native \\ leguminous plants in Llanos de Coclé, Panama \\ Avaliação do rendimento seco de Brachiaria humidicola associado a leguminosas \\ nativas em Llanos de Coclé, Panamá

Kentaro Tomita1
tomiken30@hotmail.com
Esteban Arosemena Jaén ${ }^{2}$
arosemenaestejaen@gmail.com

${ }^{1}$ Departamento de Desarrollo económica y Gestión ambiental, Gobierno Provincial de Imbabura, Ecuador. (Voluntario Senior de Agencia de Cooperación Internacional del Japón: JICA). (PreInvestigador principal. Sub- Centro Pacifico Marciaga del IDIAP [Instituto de Investigación Agropecuaria de Panamá] en El Coco y Voluntario Senior de JICA), Japón

${ }^{2}$ Investigador. Sub-Centro Pacifico Marciaga del IDIAP en El Coco, Panamá

Artículo recibido actubre 2019, arbitrado noviembre 2019 y publicado en enero 2020

\section{RESUMEN}

Se trata de un estudio en gran parte de los suelos del Arco Seco de la República de Panamá en donde han sido identificados como suelos degradados, con baja fertilidad (ANAM, 2004) por la deforestación a través del largo plazo. La finalidad fue establecer un sistema sostenible de la producción del pasto con bajos insumos en la tierra usada. Para ello se realizó el experimento después de corte de la Brachiaria humidicola viva, se preparó como un bloque experimental fue de $3 \mathrm{~m}$ por $5 \mathrm{~m}$ y totalmente, 6 tratamientos (comparación de manera de la fertilización periódica para recuperar el mismo pasto) al azar con 4 repeticiones (24 bloques) en la finca experimental de El Coco, Coclé. La duración del experimento se empezó del día 13 de agosto al día 2 de octubre del 2007, y la duración del cultivo de la Brachiaria humidicola fue de los 50 días, al alcanzar $5 \mathrm{~cm}$ de la altura, se realizó el corte.

Palabras clave: Plantas leguminosas nativas; Brachiaria humidicola; evaluación; rendimiento

\begin{abstract}
This is a study in large part of the soils of the Arco Seco of the Republic of Panama where they have been identified as degraded soils, with low fertility (ANAM, 2004) due to deforestation over the long term. The purpose was to establish a sustainable system of grass production with low inputs in the used land. For this, the experiment was performed after cutting the live Brachiaria humidicola, it was prepared as an experimental block, it was $3 \mathrm{~m}$ by $5 \mathrm{~m}$ and totally, 6 treatments (comparison in the way of periodic fertilization to recover the same grass) at random with 4 repetitions (24 blocks) in the experimental farm of El Coco, Coclé. The duration of the experiment began from August 13 to October 2, 2007, and the duration of cultivation of the Brachiaria humidicola was 50 days, when reaching $5 \mathrm{~cm}$ in height, the cut was made.
\end{abstract}

Key words: Native leguminous plants; Brachiaria humidicola; evaluation; performance 


\section{RESUMO}

Este é um estudo em grande parte dos solos do Arco Seco da República do Panamá, onde foram identificados como solos degradados, com baixa fertilidade (ANAM, 2004) devido ao desmatamento a longo prazo. 0 objetivo era estabelecer um sistema sustentável de produção de gramíneas com baixos insumos nas terras utilizadas. Para isso, o experimento foi realizado após o corte da Brachiaria humidicola viva, foi preparado como um bloco experimental, de $3 \mathrm{~m}$ por $5 \mathrm{~m}$ e totalmente, 6 tratamentos (comparação na forma de fertilização periódica para recuperar a mesma grama) aleatoriamente com 4 repetições (24 blocos) na fazenda experimental de El Coco, Coclé. A duração do experimento teve início no período de 13 de agosto a 2 de outubro de 2007, e a duração do cultivo da Brachiaria humidicola foi de 50 dias. Ao atingir $5 \mathrm{~cm}$ de altura, o corte foi realizado.

Palavras-Chave: Plantas leguminosas nativas; Brachiaria humidicola; avaliação; Desempenho

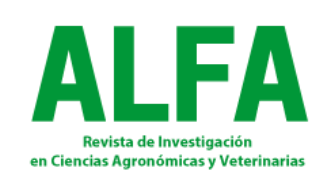

INTRODUCCIÓN

Para el rendimiento seco de la Brachiaria humidicola, fue el más alto en el T3, se considera que tuvo alta influencia de la aplicación nitrogenada adecuada. Por otra parte, también se observó la influencia por la aplicación del estiércol en los T4 y T5. Para el T5 se considera la aplicación nitrogenada elevada para el pasto gramíneo, teniendo en cuenta la cantidad aplicada del $\mathrm{N}$ químico más $1.99 \%$ del $\mathrm{N}$ como el valor contenido del estiércol con descomponerlo con el tiempo. En cambio para las leguminosas nativas totales, se observó alto rendimiento seco en los T2 y T4, los valores fueron de 1139.6 y $976.0 \mathrm{~kg} / \mathrm{ha}$, respectivamente, mientras que para el T3 $443.9 \mathrm{~kg} / \mathrm{ha}$. Para las leguminosas (Especialmente, el Desmodium desmodium), se considera que el crecimiento fue vigoroso sin la aplicación nitrogenada química, teniendo en cuenta el capaz de la fijación biológica del $\mathrm{N}$ en el aire por Rhizobio puesto a la raíz de las plantas.

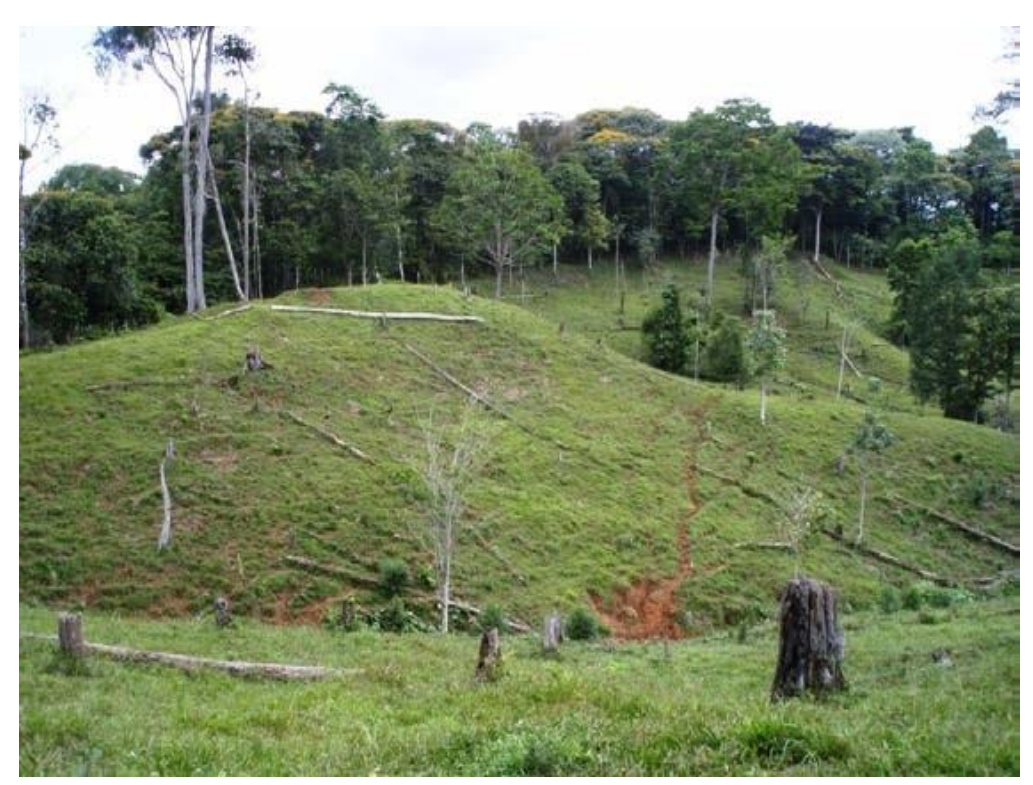

Foto 1. Condición del desmonte de árboles nativos en la región de Cordillera Central, Cocle cito, Provincia de Colón, Panama. (Foto, Tomita, 2007). 
Gran parte de los suelos del Arco Seco de la República de Panamá han sido identificados como suelos degradados, con baja fertilidad (ANAM, 2004) por la deforestación a través del largo plazo. La degradación de estos suelos, tiene su génesis desde la época Colonial por el uso del fuego en el sistema de roza y/o el sobrepastoreo.

He igualmente, campesinos panameños realizaron corte de árboles nativos para explotar ganadería, y se la avanza en la cordillera central del país (ver la Foto 1).

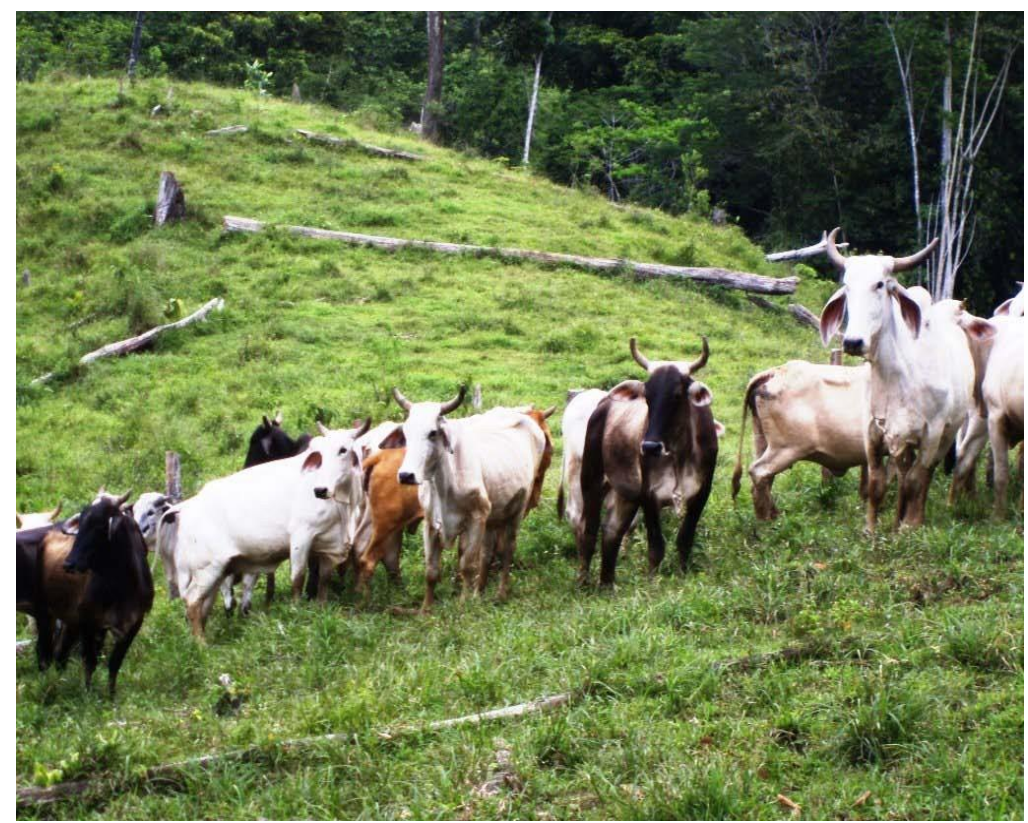

Foto 2. Ganados criados después de deforestación en la misma región con la Foto №1. (Foto, Tomita, 2007)

Las Fotos 1 y 2 muestran la condición del desmonte de árboles nativos con ganados criados en la región de Cordillera Central en Panamá. Entienden que se avanzó la deforestación en la región cordillera central en Panamá para explotar nueva pastura con crear animales domésticos, lo que significa es que: a). Avanzar destrucción ambiental; b). Contaminar los ríos cerca de la región por erosión hídrica del suelo; c). Deteriorar la característica física-química-biológica del suelo, y Degradar la producción agropecuaria sostenible, entre otros.

En realidad, se evidencia que se puede mantener la fertilidad del suelo durante alrededor de 2 o 3 años después de desmonte y quema, por lo que los campesinos realizan más explotación con el fin de buscar nueva tierra para ganados criados de nuevo. Por lo que se repita mala circulación, los autores muestran ejemplos en las Figuras 1 y 2, respectivamente. 


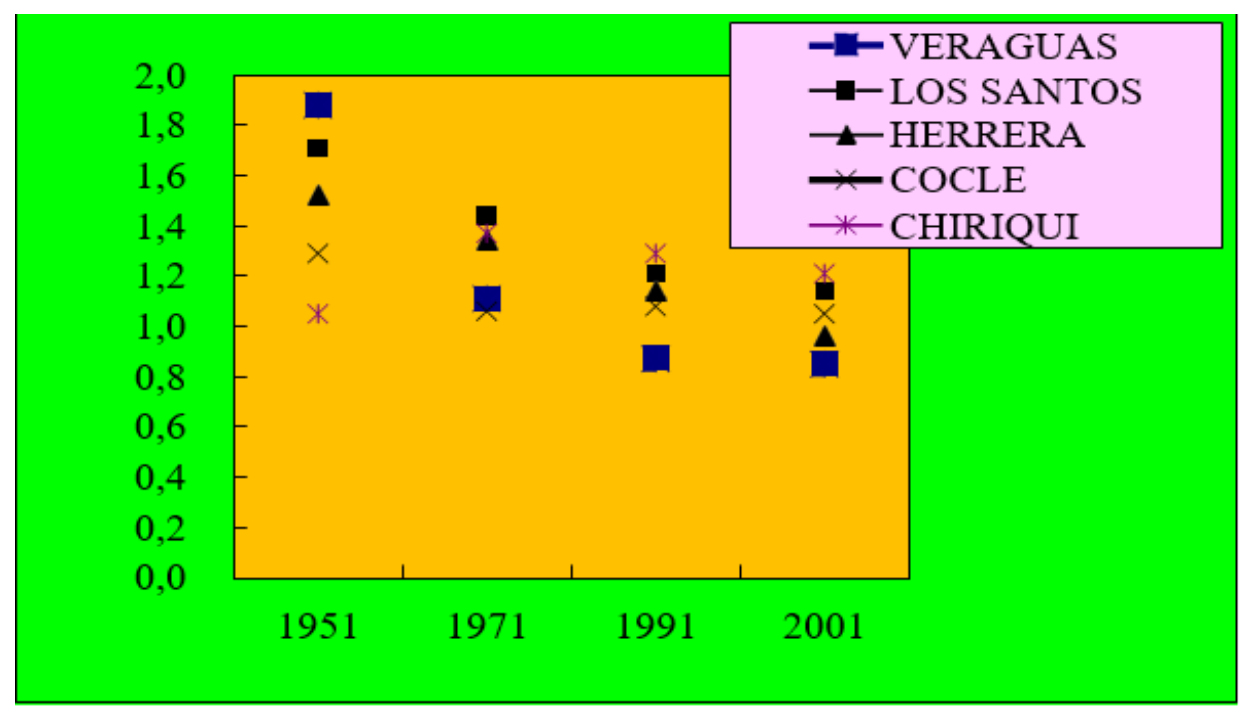

Figura 1. Dinámica de la carga animal en las provincias representativas en Panamá. (Fuente: Estadística y Censo, 1951-1991)

La Figura 1 muestra la dinámica de la carga animal por hectárea en las provincias representativas en Panamá. De la Figura, se entienden que el valor de la carga animal desapareció con el tiempo. Se considera que la fertilidad del suelo degradaron por el sobrepastoreo, especialmente, el valor en la provincia de Veraguas dentro de los años 1991 a 2001 fue el más bajo de otros porque se considera que se ocupa mucho suelo Ultisol con baja fertilidad y alto contenido del Al intercambiable. (Ávila, y Pasto, 1989).

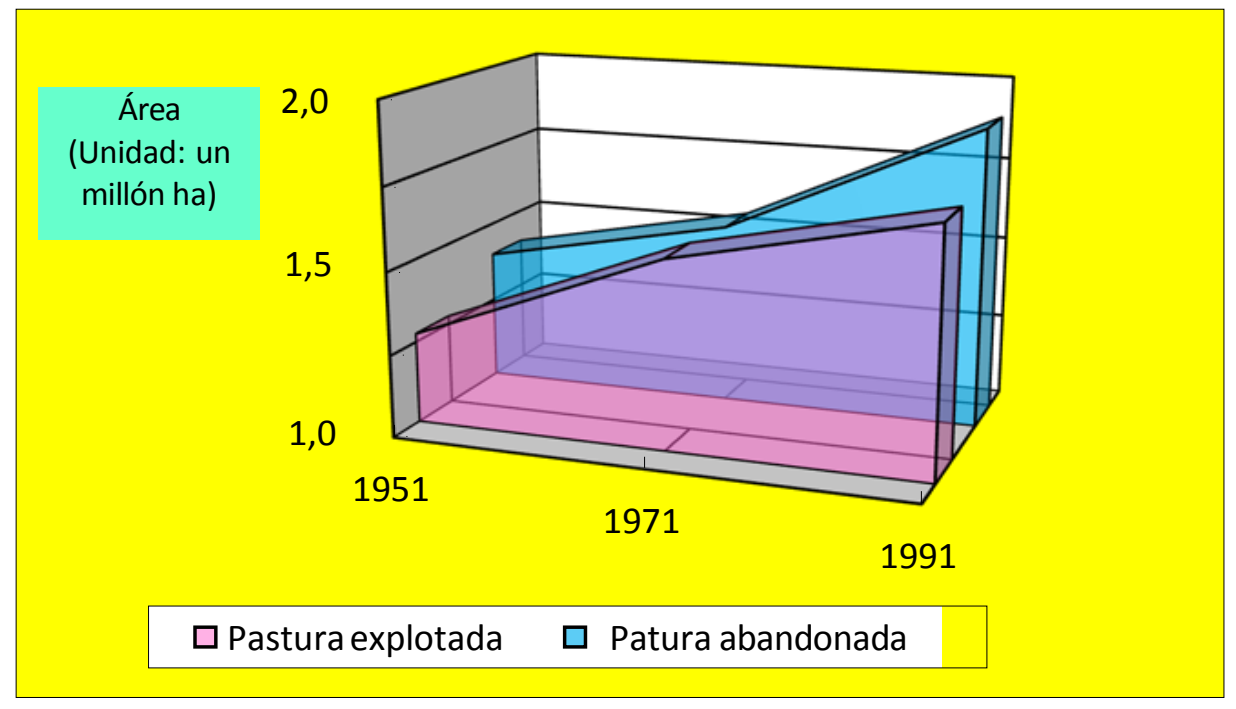

Figura 2. Dinámica del aumento de pasturas explotada y abandonada en Panamá. (Fuente: Estadística y Censo ,1991.) 
Según del investigador de Instituto Smythsonian de investigaciones Tropicales, Stanley Heckadon, (1984) "en Panamá perdemos aproximadamente 40 mil hectáreas anualmente, y en algunos años se han perdido 80 mil hectáreas, y esto se agrava por la quema de los potreros".

De las arribas mencionadas, para recuperar y/o mantener la fertilidad de los suelos, es necesario establecer un sistema sostenible de la producción del pasto con bajos insumos en la tierra usada e igualmente, se debe prevenir más desmonte y quema en la región de bosques en el país. Incluye no sólo en el Panamá sino también en todos los países de América Latina para este problema.

En el experimento, con el objeto de evaluar, en Brachiaria humidicola asociada con leguminosas nativas bajo corte, los aportes de las leguminosas y el efecto de diferentes alternativas de fertilización sobre la producción de nitrógeno de la pastura, se conduce esta actividad en el Sub-Centro Pacifico Marciaga del Instituto de Investigación Agropecuaria de Panamá (IDIAP), (Latitud norte: $8^{\circ} 25^{\prime} 00^{\prime \prime}$, Longitud oeste: $80^{\circ} 21^{\prime} 10^{\prime \prime}$, sobre un suelo clasificado en la familia fino, mezclado, isohipertérmico, Aeric Tropaquept según manifiesta Jaramillo, (1991). El clima del sitio se caracteriza por ser tropical húmedo, con promedio de 1,480 $\mathrm{mm}$ de precipitación al año, con una temperatura promedio que oscila entre 20 y $35^{\circ} \mathrm{C}$.), ubicado en Los Llanos de Coclé en la provincia de Coclé, Panamá (ver la Figura 3).

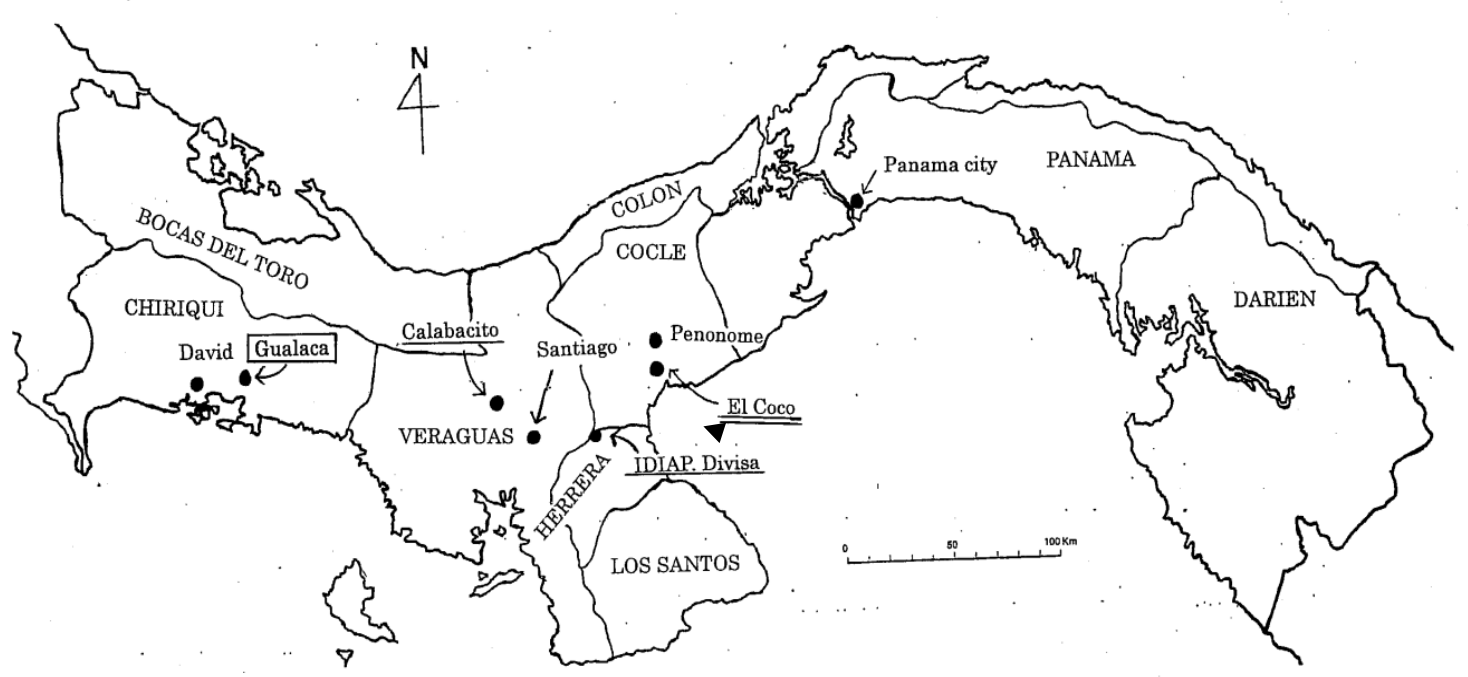

Figura 3. Sitio de la Finca Experimental de El Coco en el Subcentro Pacifico Marciaga del IDIAP en la Provincia de Coclé. (Fuente: Dibujo por el Dr. Tomita. 2009.)

MATERIALES Y MÉTODOS

\section{Tratamientos mostrados}

Se realizó el experimento después de corte de la Brachiaria humidicola viva, se preparó como un bloque experimental fue de $3 \mathrm{~m}$ por $5 \mathrm{~m}$ y totalmente, 6 tratamientos al azar con 4 repeticiones (24 bloques) en la finca experimental de El Coco, Coclé. 
- Tratamiento 1 (T1): Testigo (Planta nativa leguminosa, solamente).

- Tratamiento 2 (T2): Abonoquímicos ${ }^{* 1}+$ planta nativa leguminosa.

- Tratamiento 3 (T3): Abonoquímicos+ Urea*2 $^{*}$ planta nativa leguminosa.

- Tratamiento 4 (T4): Abonoquímicos+ Estiércol*3 + planta nativa leguminosa.

- Tratamiento 5 (T5): Abonoquímicos+ Urea+ Estiércol+ planta nativa leguminosa.

- Tratamiento 6 (T6): Abonoquímicos.

${ }^{*} 1_{\mathrm{Se}}$ aplicaron $33 \mathrm{~kg} / \mathrm{ha}, \quad 47.7 \mathrm{~kg} / \mathrm{ha}$, $15.3 \mathrm{~kg} /$ ha y $25.7 \mathrm{~kg} /$ ha como superfosfato toriple, cloruro de potasio, cal agrícola y sulfomag en los tratamientos 2 y 3, respectivamente. Por otra parte, se aplicaron $19.1 \mathrm{~kg} / \mathrm{ha}, 24.3 \mathrm{~kg} / \mathrm{ha}$ y $12.3 \mathrm{~kg}$ (las cantidades fueron mitad del tratamientos 2 y 3) como el superfosfato triple, cloruro de potasio y sulfomag, respectivamente ya que se aplicó las vacunas en los tratamientos 4 y 5 (para la cal 0t/ha).

En todos bloques se aplicó 28g del molibdato de amonio con agua en el pulverizador. ${ }^{*} 2$ La cantidad aplicada de la res fue de $69.3 \mathrm{~kg} / \mathrm{ha}$ y $28.7 \mathrm{~kg} / \mathrm{ha}$ en los tratamientos 3 y 5 , respectivamente. ${ }^{*} 3 \mathrm{La}$ cantidad aplicada del estiércol fue de $2000 \mathrm{~kg} / \mathrm{ha}$ en los tratamientos 4 y 5 .

\section{Duración del experimento}

La duración del experimento se empezó del día 13 de agosto al día 2 de octubre del 2007, y la duración del cultivo de la Brachiaria humidicola fue de los 50 días, al alcanzar $5 \mathrm{~cm}$ de la altura, se realizó el corte, bajo la metodología de Díaz-Romeú, y Hunter, (1978).

\section{Análisis del suelo, estiércol y tejido vegetal}

Para el análisis del suelo, estiércol con excepción del $\mathrm{N}$ y tejido vegetal, el autor se los pidió al Laboratorio de suelos del IDIAP donde está en Divisa, ubicado en la provincia de Veraguas, por otra parte, para el análisis del $\mathrm{N}$ del estiércol al laboratorio del Instituto ganadero del IDIAP donde está en Gualaca, ubicado en la provincia de Chiriquí.

\section{RESULTADOS Y DISCUSIÓN}

\section{Análisis del suelo después del corte de Brachiaria humidicola viva}

La Tabla 1 muestra el análisis del suelo después del corte de Brachiaria humidicola. Se observó bajo contenido de P, K, Ca, Mg y M.O. como la característica química, para la física fue arenoso. Por eso, se considera que fue el suelo empeorado. Esta condición será parecida con la tierra abandonada $\mathrm{y} / \mathrm{o}$ degradada por el sobrepastoreo, al establecer un sistema sostenible de la producción, se podrá contribuir al manejo de la tierra.

\section{Análisis del estiércol de una finca de ganadería}

La Tabla 2 muestra el análisis del estiércol de una fina de ganadería cerca de la finca experimental del Subcentral Pacifico Marciaga del IDIAP. Se observó que el valor del N fue de $1.99 \%$, y alto contenido del Mn y Fe. Se considera que hubo influencia de la cantidad quedad de los dos elementos micros en la finca.

Por otra parte por observación del 8.2 como $\mathrm{pH}$, se podrá utilizar un material como corrección de acidez del suelo. 
Tabla 1. Análisis del suelo después del corte de Brachiaria humidicola viva

\begin{tabular}{|c|c|c|c|c|c|c|c|c|c|c|}
\hline \multicolumn{3}{|c|}{ Granulometría } & \multirow{3}{*}{$\begin{array}{c}\mathrm{pH} \\
\mathrm{H}_{2} \mathrm{O} \\
1 ; 1\end{array}$} & \multicolumn{2}{|c|}{ Disponible } & \multicolumn{3}{|c|}{ Intercambiables } & \multirow[t]{2}{*}{ CICE } & \multirow[t]{2}{*}{ M.O. } \\
\hline Arena & Limo & Arcilla & & $P$ & $\mathrm{~K}$ & $\mathrm{Ca}$ & $\mathrm{Mg}$ & $\mathrm{Al}$ & & \\
\hline \multicolumn{3}{|c|}{$(\%)$} & & \multicolumn{2}{|c|}{$(\mathrm{mg} / \mathrm{L})$} & \multicolumn{4}{|c|}{$\mathrm{cmol} / \mathrm{kg}$} & $(\%)$ \\
\hline 62,0 & 22,0 & 16,0 & 5,5 & 2,0 & 39,0 & 1,6 & 0,7 & 0,1 & 2,4 & 0,80 \\
\hline \multicolumn{4}{|c|}{ Elementos menores } & & & & & & & \\
\hline $\mathrm{Mn}$ & $\mathrm{Fe}$ & $\mathrm{Zn}$ & $\mathrm{Cu}$ & & & & & & & \\
\hline \multicolumn{4}{|c|}{$(\mathrm{mg} / \mathrm{L})$} & & & & & & & \\
\hline 90,0 & 52,0 & 1,0 & 1,0 & & & & & & & \\
\hline
\end{tabular}

\section{Análisis realizados en el Laboratorio de suelos del IDIAP en Divisa}

Métodos analíticos: $\mathrm{pH}$ en agua (1:1); $\mathrm{P}$, $\mathrm{K}, \mathrm{Mn}, \mathrm{Fe}, \mathrm{Zn}$ y $\mathrm{Cu}=$ Extractor Mehlich 1 $\left(0.05 \mathrm{M} \mathrm{HCl}+0.0125 \mathrm{M} \mathrm{H}_{2} \mathrm{SO}_{4}\right) ; \mathrm{Ca}, \mathrm{Mg} \mathrm{y} \mathrm{Al}=$ Extractor $\mathrm{KCl}$ al 1M; M.O. = Materia Orgánica (Walkey-Black modificado); Análisis física = Bouyoucos.

\section{Análisis del estiércol de una finca de ganadería}

La Tabla 2 muestra el análisis del

Tabla 2. Análisis del estiércol de una finca ganadería

\begin{tabular}{|c|c|c|c|c|c|c|c|c|c|}
\hline \multirow{2}{*}{ pH } & $\mathbf{N}$ & $\mathbf{P}_{2} \mathrm{O}_{5}$ & $\mathbf{K}_{2} \mathbf{O}$ & $\mathrm{CaO}$ & MgO & Mn & $\mathbf{F e}$ & $\mathrm{Cu}$ & Zn \\
\hline & \multicolumn{5}{|c|}{$\%$} & \multicolumn{4}{|c|}{$\mathrm{mg} / \mathrm{kg}$} \\
\hline 8.2 & 1.99 & 0.42 & 1.22 & 5.29 & 1.88 & 1000 & 4000 & 90 & 230 \\
\hline
\end{tabular}

\section{Rendimiento seco para cada pasto $\mathrm{y}$ corte}

La Foto 3 muestra el tratamiento de Brachiaria humidicola, y la Figura 4 muestra el rendimiento seco para cada pasto y corte. En el experimento contiene la evaluación de Cyperacea como maleza gramínea. Para el rendimiento seco de la Brachiaria humidicola, fue el más alto en el T3, se considera que tuvo alta influencia de la aplicación nitrogenada adecuada. Por otra parte, también se observó estiércol de una fina de ganadería cerca de la finca experimental del Subcentro Pacifico Marciaga del IDIAP. Se observó que el valor del $\mathrm{N}$ fue de $1.99 \%$, y alto contenido del Mn y Fe. Se considera que hubo influencia de la cantidad quedad de los dos elementos micros en la finca.

Por otra parte; por observación del 8.2 como $\mathrm{pH}$, se podrá utilizar un material como corrección de acidez del suelo. 


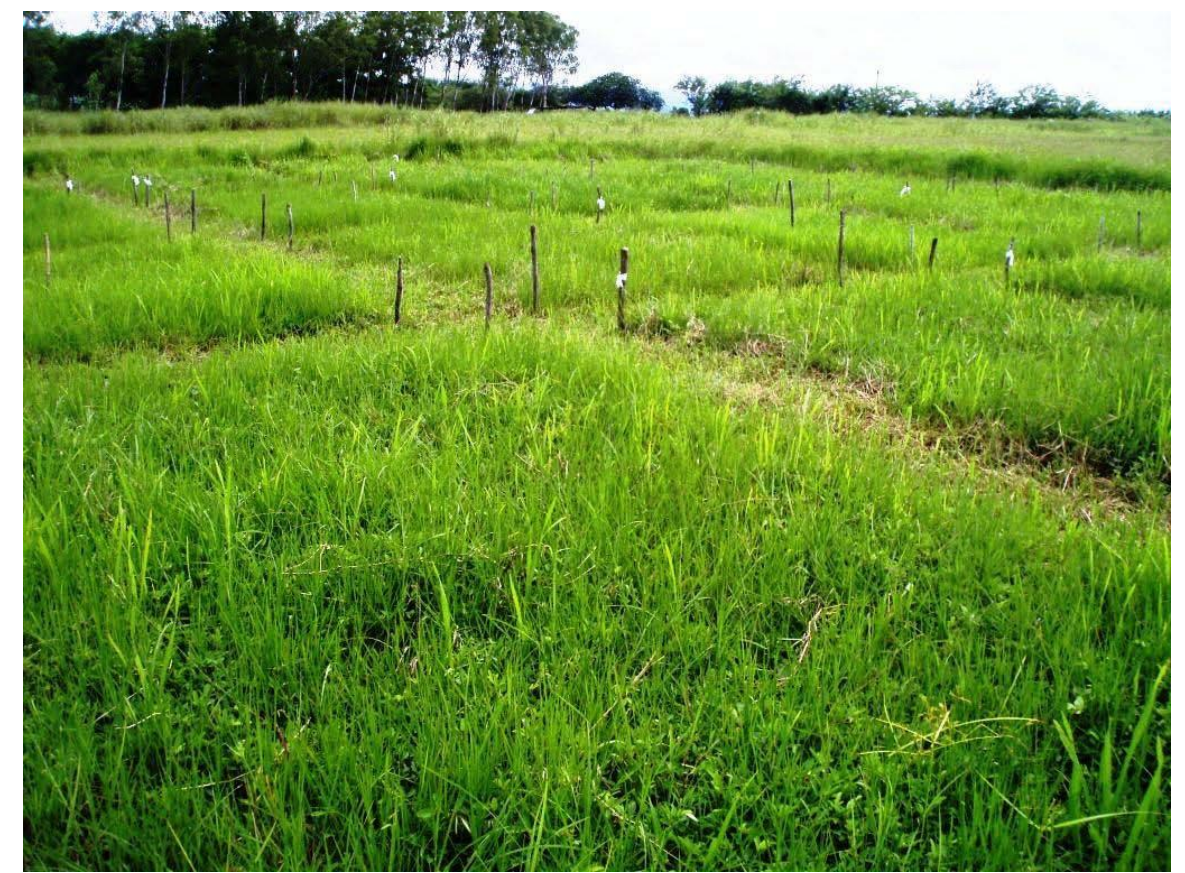

Foto 3. Tratamiento de Brachiaria humidicola, 2007. (Foto, Tomita, 2007)

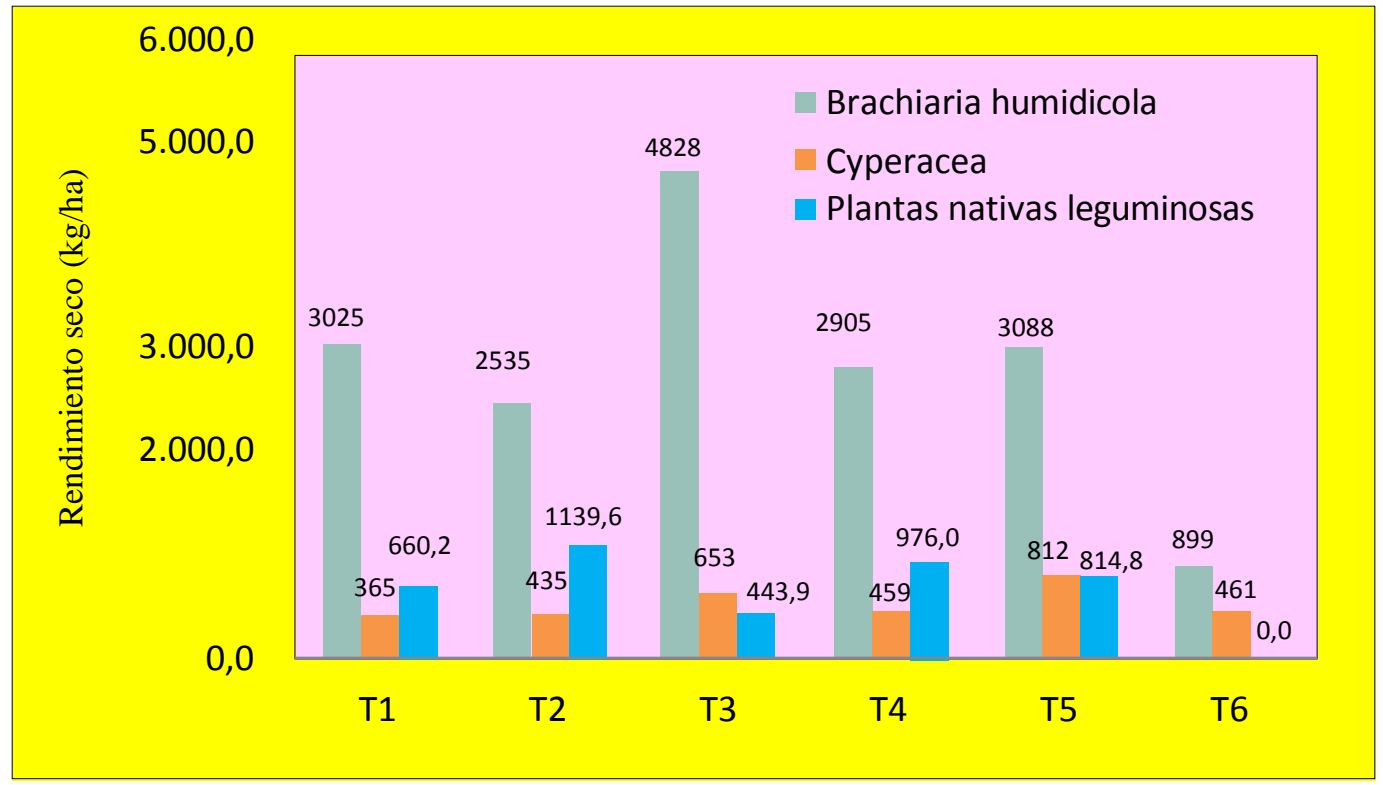

Figura 4. Rendimiento seco para cada pasto y corte 
- $\quad$ PS: T1 = Tratamiento 1: Testigo (Planta nativa leguminosa, solamente) $\mathrm{T} 2=$ Tratamiento 2: Abonoquímicos+ planta nativa leguminosa

- $\mathrm{T} 3$ = Tratamiento 3: Abonoquímicos+ Urea+ planta nativa leguminosa

- $\mathrm{T} 4$ = Tratamiento 4: Abonoquímicos+ Estiércol+ planta nativa leguminosa

- $\mathrm{T} 5$ = Tratamiento 5: Abonoquímicos+ Urea+ Estiércol+ planta nativa leguminosa $\mathrm{T} 6=$ Tratamiento 6 : Abonoquímicos

Por otra parte; para las leguminosas nativas totales, se observó alto rendimiento seco en los T2 y $\mathrm{T} 4$, los valores fueron de 1139.6 y $976.0 \mathrm{~kg} / \mathrm{ha}$, respectivamente, mientras que para el T3 $443.9 \mathrm{~kg} / \mathrm{ha}$. Para las leguminosas, se considera que el crecimiento fue vigoroso sin la aplicación nitrogenada química, teniendo en cuenta el capaz de la fijación biológica del $\mathrm{N}$ en el aire por Rhizobio puesto a la raíz de las plantas.

Para la Cyperacea, se observó alto rendimiento seco en los T3 y $\mathrm{T} 5, \mathrm{y}$ se considera que haría influencia de la aplicación nitrogenada química.

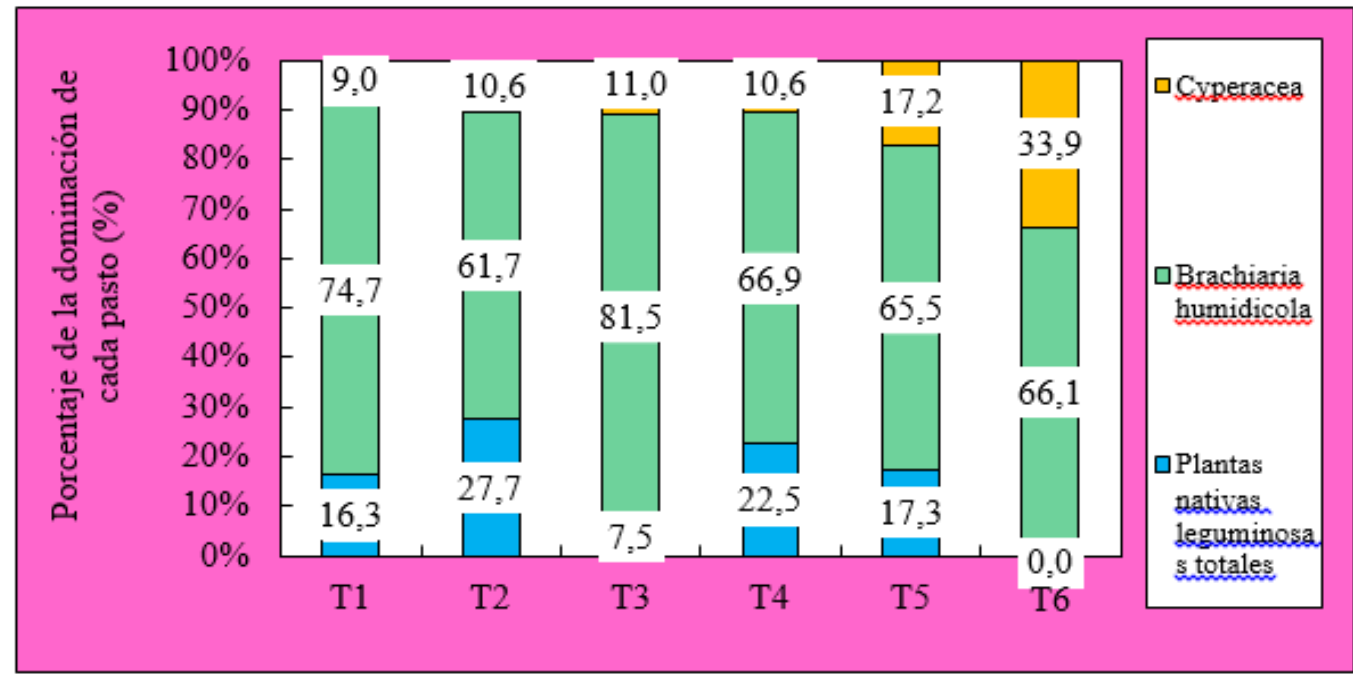

Figura 5. Proporción de Cyperacea, Brachiaria humidicola y plantas leguminosas totales en cada tratamiento.

\section{Proporción de Cyperacea, Brachiaria humidicola y Plantas leguminosas totales}

La Figura 5 muestra la proporción de Cyperacea, Brachiartia humidicola y Plantas leguminosas totales en cada tratamiento. Relativamente, la proporción de la Brachiaria humidicola fue el más alta proporción de otras plantas en todos los tratamientos, especialmente la proporción de la Brachiaria humidicola en el T3 fue el más alto de la del mismo pasto gramíneo en otros tratamientos porque hubo alta influencia de la aplicación nitorgenada química.
Por otra parte, para las plantas legumonosas totales, se las observó alta proporción en los T2 y T4 porque no se aplicó el abono nitrogenado químico. Se considera que para Rhizobio la actividad sería aumentada en la condición que no se aplicara el abono nitrogenado. Por otra parte, se observó baja proporción de las leguminosas totales en el T1 en comparación con las en los T2 y T4. Se considera que la actividad del Rhyzobio se inhibió en la condición de la deficiencia del $\mathrm{P}$ en el suelo. 
Tabla 3. Dominación de cada planta leguminosa nativa (\%)

\begin{tabular}{lccccc}
\hline & Desmodium & Calopogonio & Stylosanthes & Otras & Total \\
\hline T1 & 17.65 & 11.76 & 68.63 & 1.96 & 100 \\
T2 & 65.97 & 29.32 & 0 & 4.71 & 100 \\
T3 & 31.25 & 68.75 & 0 & 0 & 100 \\
T4 & 51.96 & 37.25 & 10.79 & 0 & 100 \\
T5 & 20.69 & 79.31 & 0 & 0 & 100 \\
\hline Total & 187.52 & 226.39 & 79.42 & 6.67 & 500 \\
\hline
\end{tabular}

PS: Desmodium = Desmodium barbatum, Calopogonio = Calpogonium mucunoides, Stylosanthes = Stylosanthes spp. $\mathrm{y}$ como otas, se incluyen Alysicarpus, Centrocema y Asechinemene.

La Tabla 3 muestra la dominación de cada planta leguminosa nativa en cada tratamiento. En cada tratamiento, se observó distinto resultado, pero en los T2 y T4 la dominación del Desmodium fue alta, por el contrario para el Calopogonio la alta dominación en los T3 y T5. Además para el Stylosanthes alta dominación en el T1.

Se considera que para el Desmodium hubo mala influencia de la aplicación nitrogenada química y el nitrógeno con el estiércol, teniendo en cuenta poca descomposición con el $\mathrm{N}$ inorgánico liberado, por el contrario para el Calopogonio prefirió el $\mathrm{N}$ aplicado con el estiércol a la condición sin la aplicación. Para las Fotos 4 y 5, muestran Desmodium (Desmodium barbatum) y Calopogonio (Calopogonium mucunoides), respectivamente.

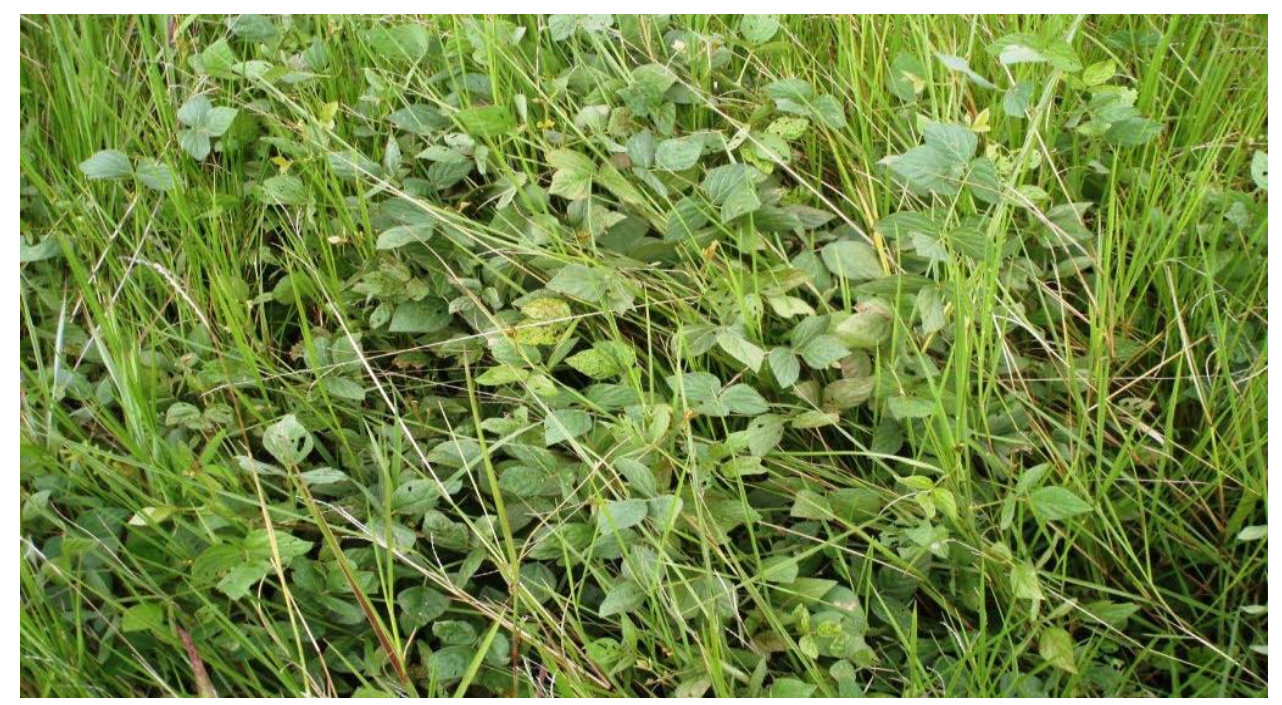

Foto 4. Desmodium (Desmodium barbatum) asociado con Brachiaria humidicola. (Foto, Tomita, 2007). 


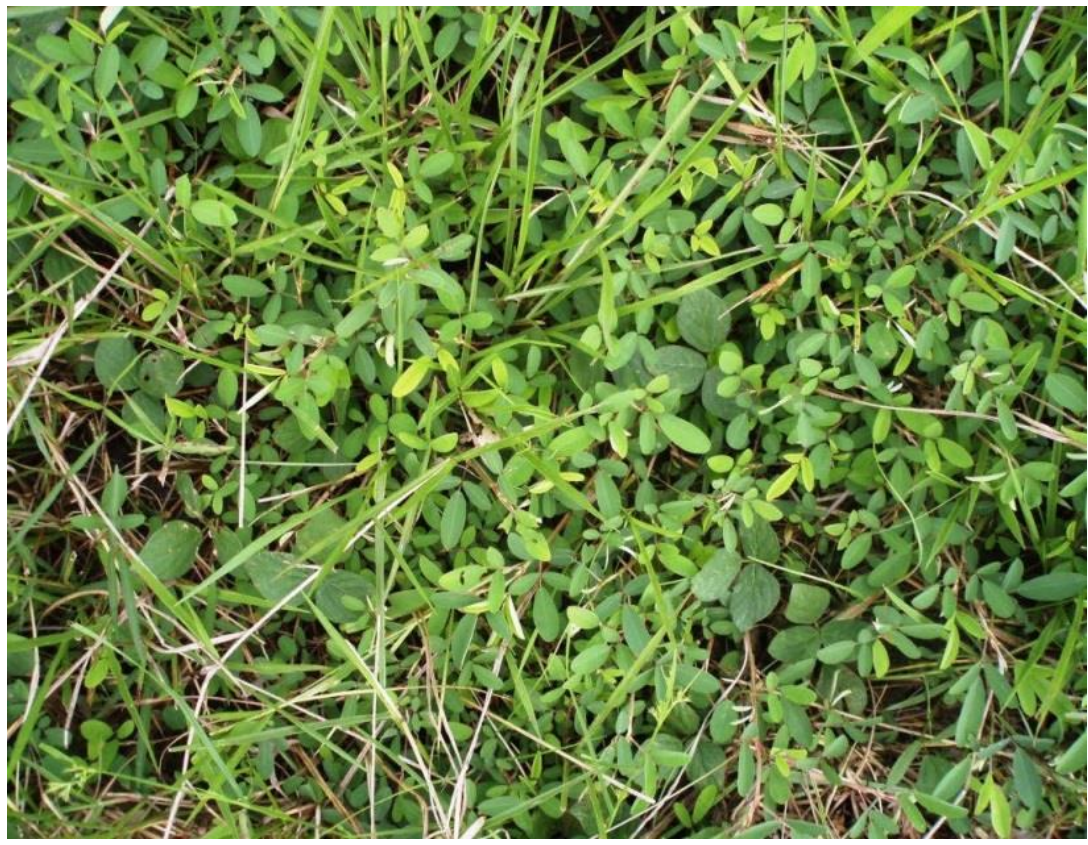

Foto 5. Calopogonio (Calopogonium mucunoides) asociado con Brachiaria humidicola. (Foto, Tomita, 2007)

\section{Cantidad absorbida de los 9 nutrientes en cada pasto y en cada tratamiento}

La Tabla No4 muestra el análisis de los 9 nutrientes en cada pasto y en cada tratamiento. Los resultados se utilizan cálculo de la cantidad absorbida de los nutrientes de cada pasto y en cada tratamiento, por lo que explica los resultados de la cantidad absorbida de cada nutrimento en las Figuras siguientes.

Tabla 4. Análisis de los 9 nutrientes en cada pasto y en cada tratamiento

\begin{tabular}{cccccccccc}
\hline & $\mathbf{N}(\%)$ & $\mathbf{P ( \% )}$ & $\mathbf{K}(\%)$ & $\mathbf{C a}(\%)$ & $\mathbf{M g}(\%)$ & $\operatorname{Mn}(\mathrm{ppm})$ & Fe(ppm) & Zn(ppm) & Cu(ppm) \\
\hline T1-Bh & 0,368 & 0,130 & 1,114 & 0,230 & 0,155 & 300 & 220 & 20 & 10 \\
T2-Bh & 0,434 & 0,165 & 1,251 & 0,240 & 0,165 & 320 & 220 & 15 & 0 \\
T3-Bh & 0,469 & 0,150 & 1,408 & 0,240 & 0,175 & 220 & 160 & 15 & 15 \\
T4-Bh & 0,399 & 0,295 & 1,553 & 0,254 & 0,190 & 260 & 125 & 30 & 15 \\
T5-Bh & 0,483 & 0,300 & 1,584 & 0,255 & 0,205 & 270 & 135 & 40 & 20 \\
T6-Bh & 0,188 & 0,120 & 1,108 & 0,220 & 0,135 & 445 & 140 & 5 & 10 \\
T1-CM & 1,148 & 0,140 & 1,447 & 0,830 & 0,260 & 170 & 150 & 20 & 0 \\
T2-CM & 0,910 & 0,200 & 1,232 & 0,915 & 0,300 & 280 & 210 & 10 & 10 \\
T3-CM & 1,337 & 0,155 & 1,271 & 0,890 & 0,340 & 220 & 150 & 20 & 5 \\
T4-CM & 1,239 & 0,265 & 1,466 & 0,920 & 0,340 & 160 & 140 & 20 & 10 \\
\hline
\end{tabular}


Evaluación del rendimiento seco de la Brachiaria humidicola asociada con plantas leguminosas nativas en Llanos de Coclé, Panamá

\begin{tabular}{cccccccccc}
\hline & $\mathbf{N}(\%)$ & $\mathbf{P ( \% )}$ & $\mathbf{K}(\%)$ & $\mathbf{C a}(\%)$ & $\mathbf{M g}(\%)$ & $\operatorname{Mn}(\mathbf{p p m})$ & $\mathrm{Fe}(\mathrm{ppm})$ & $\mathrm{Zn}(\mathrm{ppm})$ & Cu(ppm) \\
\hline T5-CM & 1,204 & 0,240 & 1,271 & 0,835 & 0,310 & 220 & 140 & 30 & 15 \\
T1-DB & 0,924 & 0,190 & 1,290 & 0,770 & 0,250 & 355 & 215 & 15 & 5 \\
T2-DB & 1,029 & 0,145 & 1,329 & 0,760 & 0,260 & 405 & 335 & 15 & 10 \\
T3-DB & 1,085 & 0,135 & 1,114 & 0,731 & 0,275 & 330 & 285 & 20 & 15 \\
T4-DB & 0,952 & 0,285 & 1,271 & 0,775 & 0,300 & 320 & 180 & 30 & 5 \\
T5-DB & 0,826 & 0,300 & 1,212 & 0,855 & 0,315 & 360 & 135 & 30 & 10 \\
T1-Sg & 0,924 & 0,250 & 1,251 & 0,980 & 0,330 & 260 & 180 & 0 & 20 \\
T2-Sg & 0 & 0 & 0 & 0 & 0 & 0 & 0 & 0 & 0 \\
T3-Sg & 0 & 0 & 0 & 0 & 0 & 0 & 0 & 0 & 0 \\
T4-Sg & 1,498 & 0,350 & 1,525 & 0,720 & 0,410 & 100 & 110 & 30 & 10 \\
T5-Sg & 0 & 0 & 0 & 0 & 0 & 0 & 0 & 0 & 0 \\
\hline
\end{tabular}

PS: $\mathrm{Bh}=$ Brachiaria humidicola, $\mathrm{CM}=$ Calopogonium mucunoides, $\mathrm{DB}=$ Desmodium barbatuma y Sg = Stylosanthes spp.

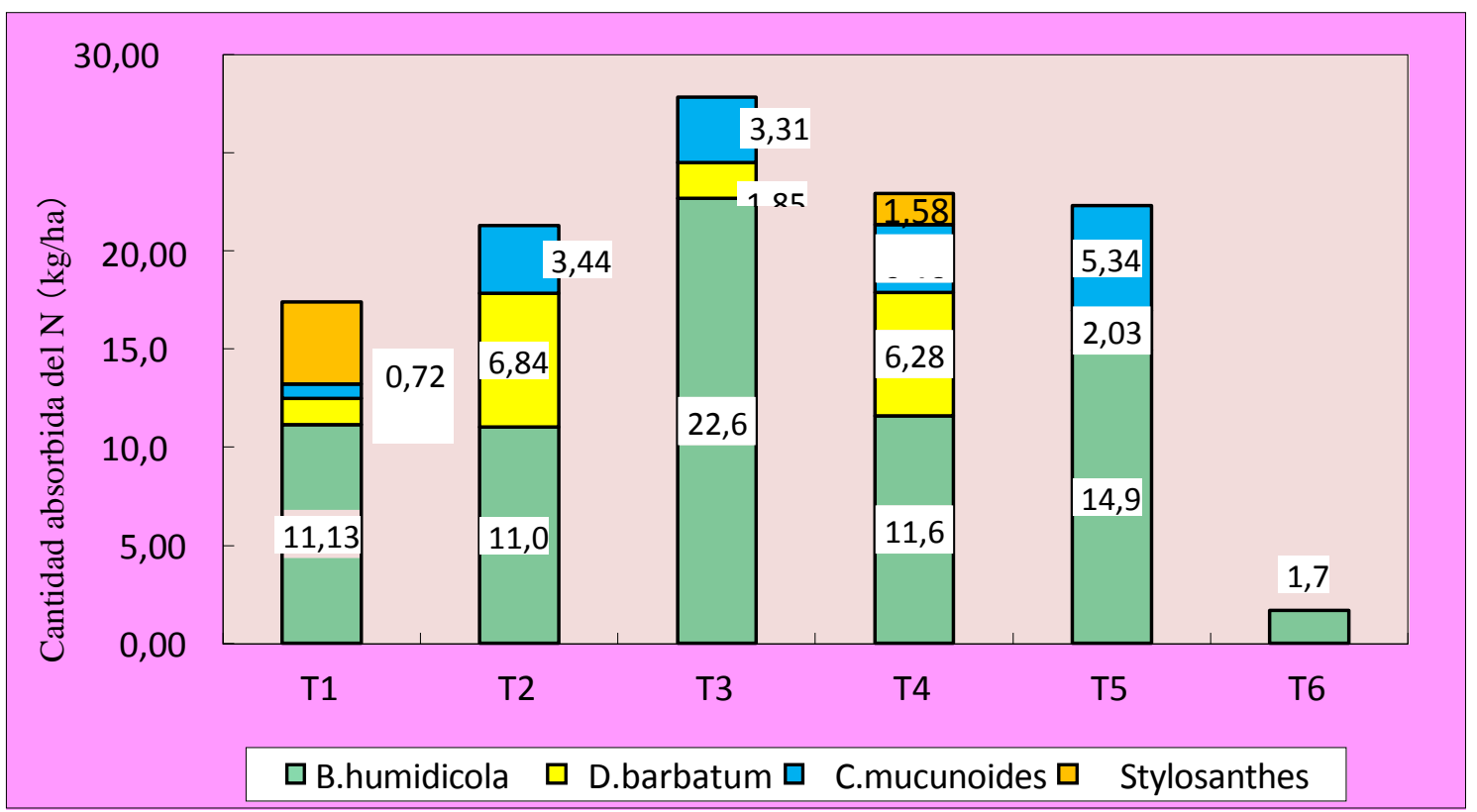

Figura 6. Cantidad absorbida del $\mathrm{N}$ en cada pasto y en cada tratamiento.

La Figura 6 muestra la cantidad absorbida del N en cada pasto y en cada tratamiento. Suma por la cantidad absorbida en el T3 fue el más alta de la suma de otros tratamientos, y se considera que hubo influencia del gran crecimiento y la absorción para la Brachiaria humidicola. Pero para el Desmodium barbatum, la Cantidad en el T3 fue más baja que la cantidad en los T2 y T4 porque era la condición sin la aplicación nitrogenada química. 
Totalmente, del punto de la vista de la suma por la cantidad del $\mathrm{N}$ (Material proteico), se pareció que los T2, T4 y T5 fueron altas adecuadas para el suministro a ganados criados, y los tratamientos tuvieron una balanza del pasto gramíneo y leguminoso en comparación con el T3. A continuación, explica la suma por la cantidad absorbida de otros 8 nutrientes en las Figuras siguientes.

La Figura 7 muestra la cantidad absorbida del $\mathrm{P}$ en cada pasto y en cada tratamiento. A diferencia de los resultados del $\mathrm{N}$, la Suma por la cantidad absorbida en el T4 y el T5 fue el más alta de la suma de otros tratamientos, y se considera que hubo influencia de quelato producido al camino de la descomposición del estiércol aplicado, previno la fijación o la adsorción del $\mathrm{P}$ al coloide del suelo. Por el contrario, en los tratamientos sin el estiércol, relativamente, la suma por la cantidad absorbida fosfatada fue baja para cada pasto, pero para el Desmodium burbatum en el T2 fue alta absorción al igual que el caso en el T4. Se considera que el poder absorbido fosfatado sería fuerte en la condición sin la aplicación nitrogenada química.

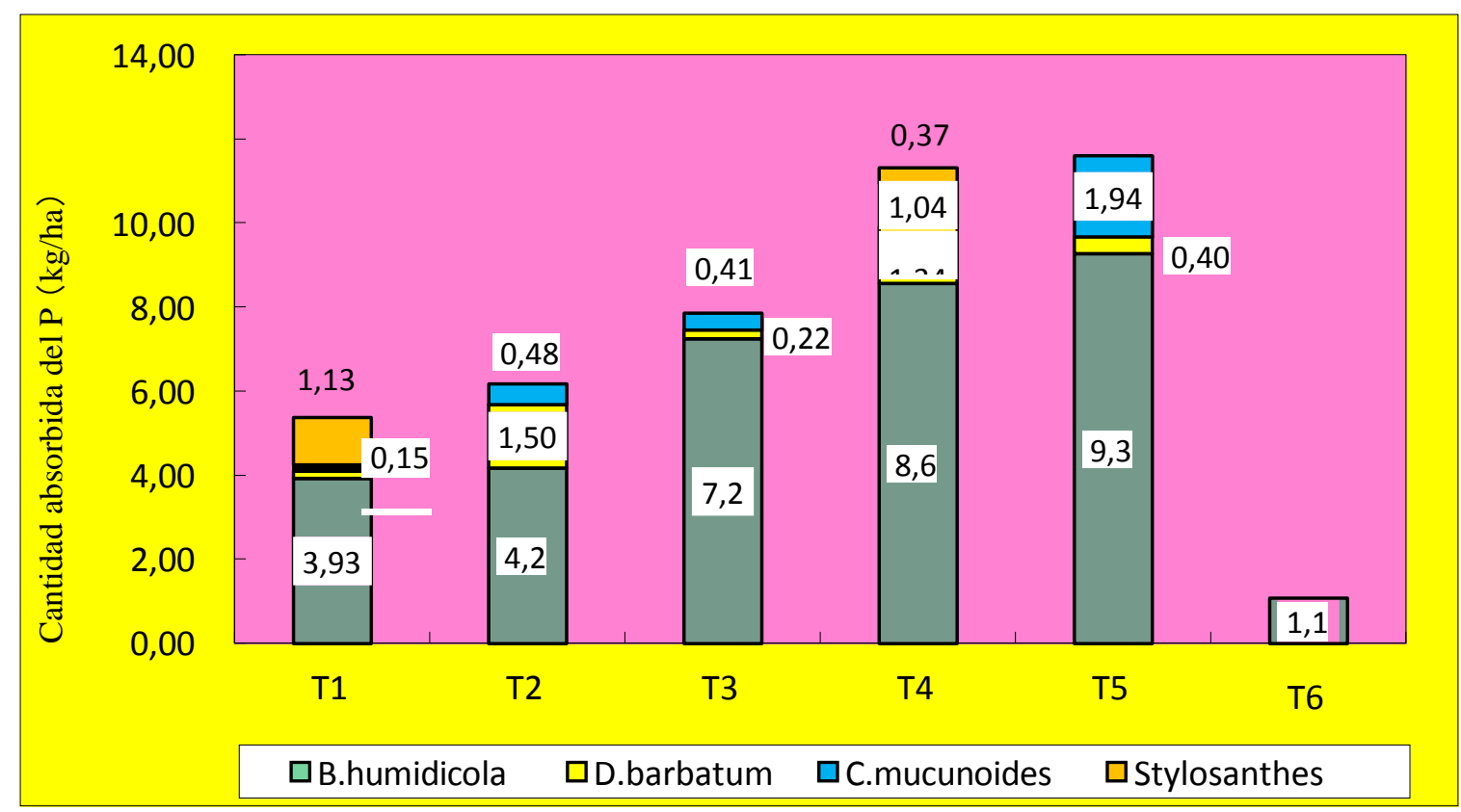

Figura 7. Cantidad absorbida del P en cada pasto y en cada tratamiento. 
Evaluación del rendimiento seco de la Brachiaria humidicola asociada con plantas leguminosas nativas en Llanos de Coclé, Panamá

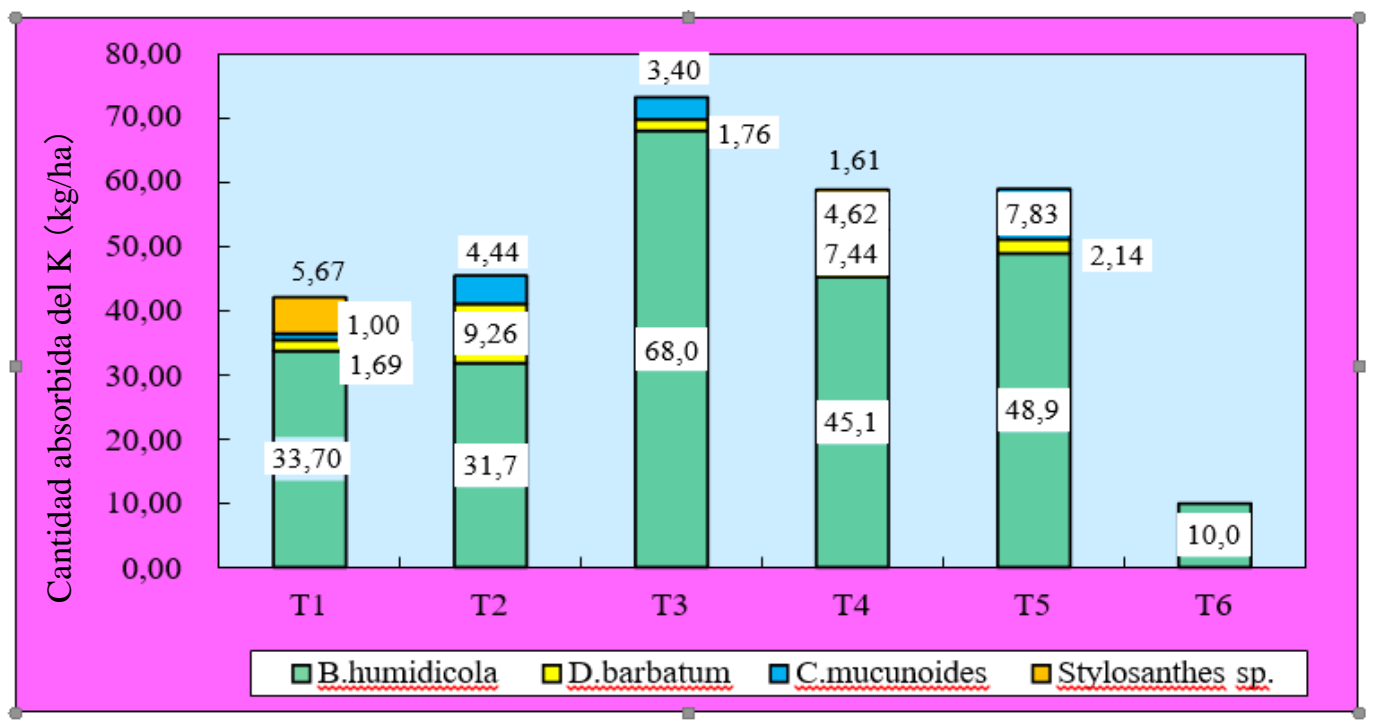

Figura 8. Cantidad absorbida del K en cada pasto y en cada tratamiento.

La Figura 8 muestra la cantidad absorbida del $\mathrm{K}$ en cada pasto y en cada tratamiento. Al igual que el caso del N, la Suma por la cantidad absorbida en el T3 fue el más alta de la suma de otros tratamientos, siguiendo para los T4 y T5 fueron el más segunda altas. Se considera que hubo influencia del estiércol aplicado, teniendo en cuenta alto contenido del K (1.22\%: ver la Tabla 2).

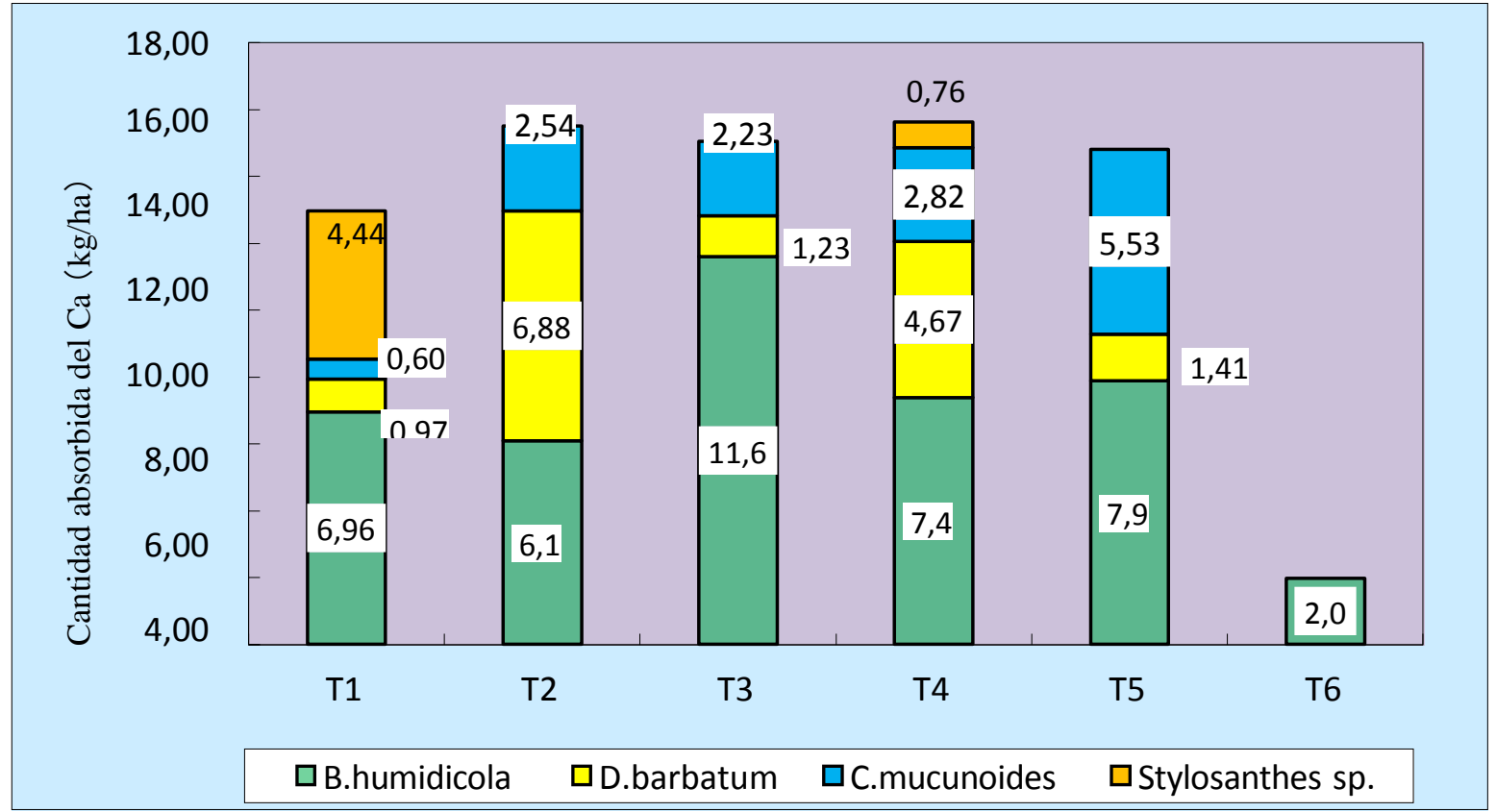

Figura 9. Cantidad absorbida del Ca en cada pasto y en cada tratamiento. 
La Figura 9 muestra la cantidad absorbida del Ca en cada pasto y en cada tratamiento. Para la suma de la cantidad absorbida, los valores en los T2, T3, T4 y T5 fueron similares, pero, para el T3, la balanza de la dominación de los pastos fue mala. Especialmente, la cantidad del Desmodium en el T2 fue más alta que la cantidad en el T4, se considera que el poder absorbida del Ca para el Desmodium barbatum fue relevante en la condición sin la aplicación del N químico ni el estiércol.

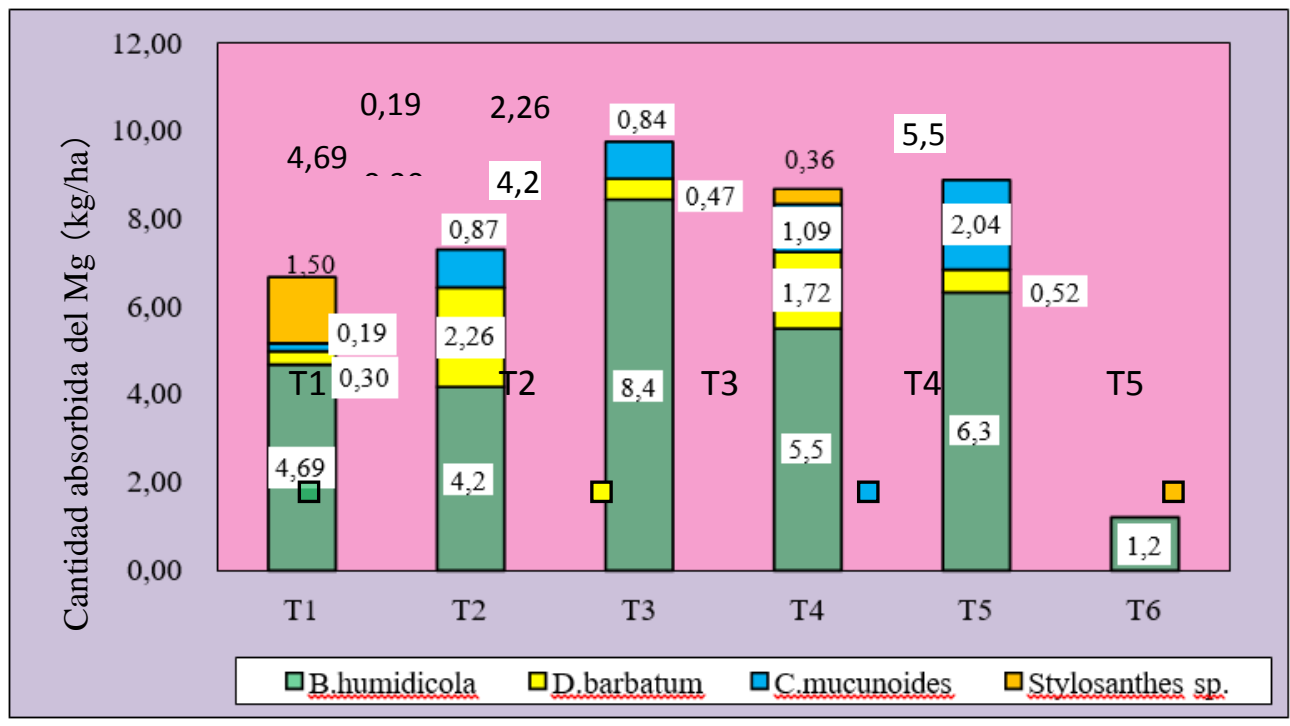

Figura 10. Cantidad absorbida del Mg en cada pasto y en cada tratamiento.

La Figura 10 muestra la cantidad absorbida del Mg en cada pasto y en cada tratamiento. A diferencia del resultado del caso del Ca, la suma en el T3 fue el más alta al igual que el caso de la suma del $\mathrm{N}$ y K, pero la balanza de cada pasto en el T3 fue mala, por otra parte para el T2 y T4 fue la buena. Especialmente, la cantidad del Desmodium barbatum en el T2 fue más alta que la cantidad en el T4, se considera que el poder absorbida del Mg para el Desmodium barbatum fue relevante en la condición sin la aplicación del $\mathrm{N}$ químico ni el estiércol al igual que el caso del Ca.

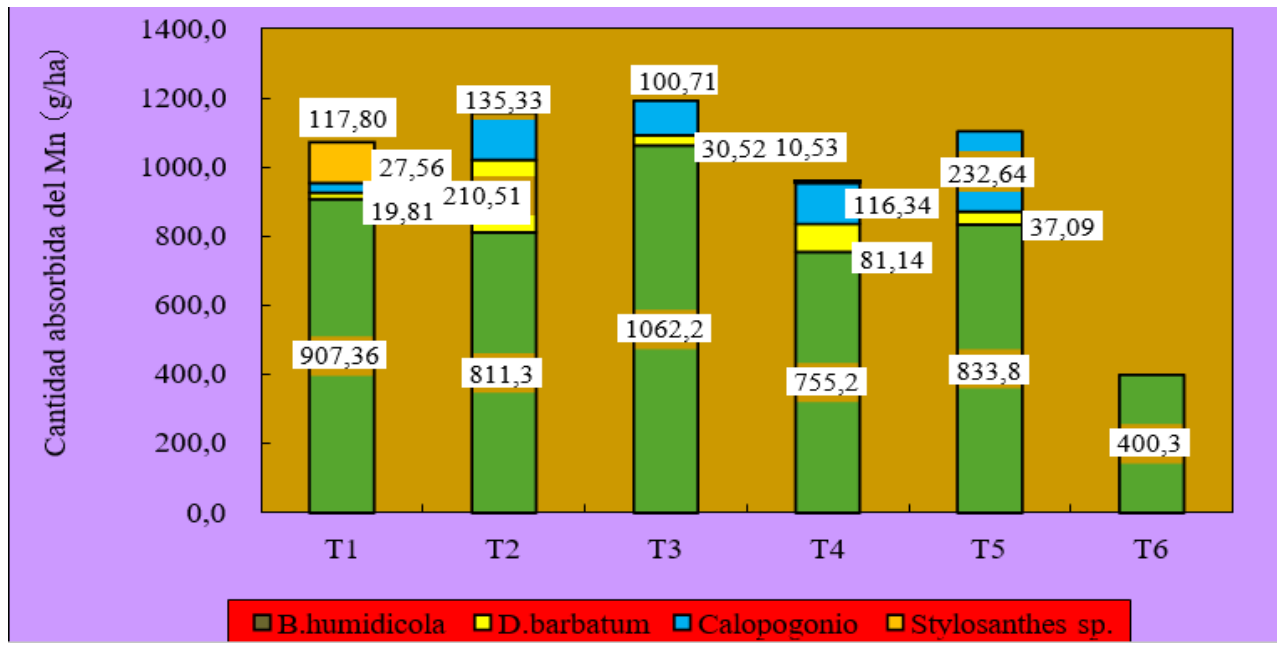

Figura 11. Cantidad absorbida del Mn en cada pasto y en cada tratamiento. Suma en el T3 fue el más alta de la suma de otros tratamientos, y se observó la misma tendencia con el caso del Ca y Mg. 


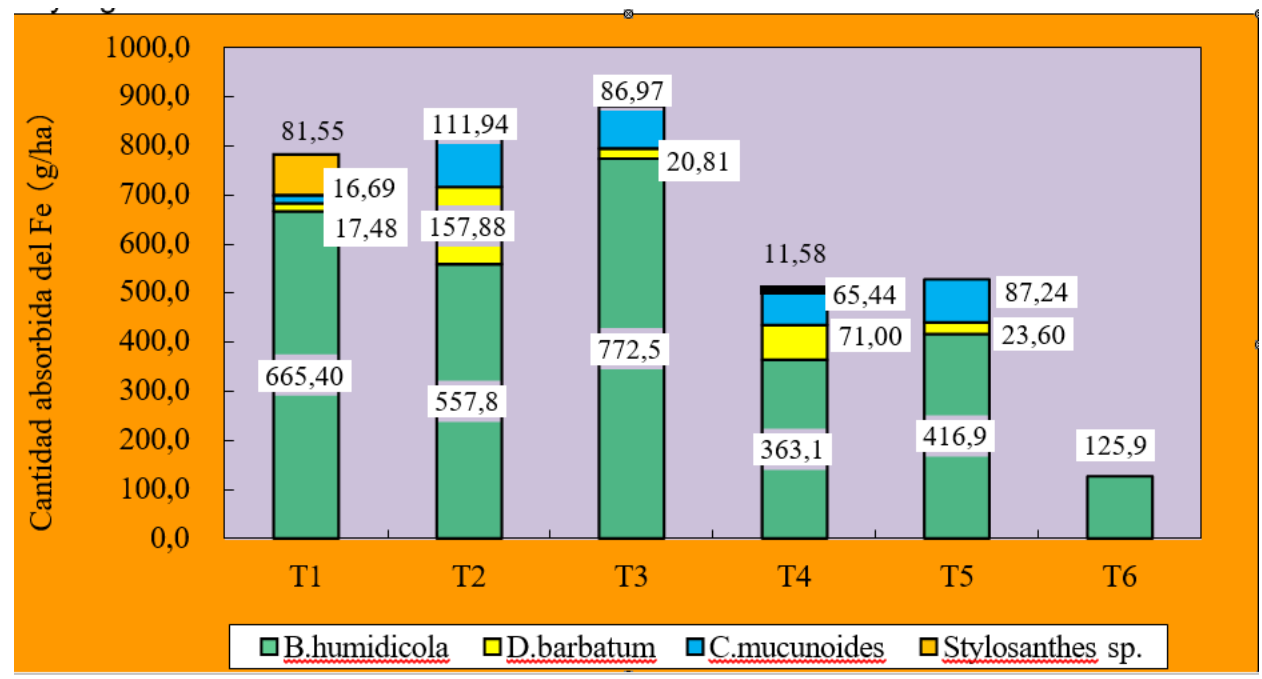

Figura 12. Cantidad absorbida del Fe en cada pasto y en cada tratamiento.

La Figura 12 muestra la cantidad absorbida del Fe en cada pasto y en cada tratamiento. Para el Fe, la suma en el T3 fue el más alta de la suma de otros tratamientos, pero. Para los T4 y T5 se observó que la suma fue más baja que la suma en los T1 a T3, la suma en el T6 fue el más baja. Especialmente, se considera que habría una influencia del estiércol aplicado porque se adsorbió el Fe en el material. Para la cantidad en el T6, se dependería el rendimiento seco de la Brachiaria humidicola sin las leguminosas, ni la aplicación de abonos químicos.

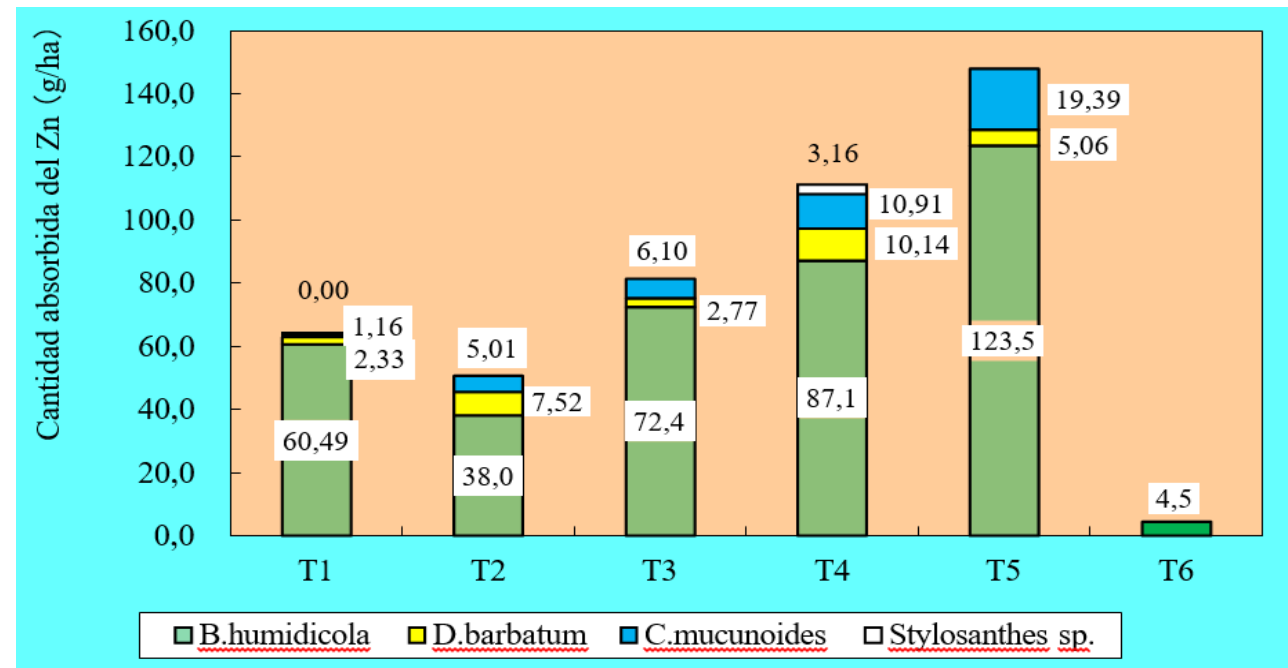

Figura 13. Cantidad absorbida del Zn en cada pasto y en cada tratamiento.

La Figura 13 muestra la cantidad absorbida del Zn en cada pasto y en cada tratamiento. Para el Zn, la suma en el T5 fue el más alta de la suma de otros tratamientos a diferencias de los resultados obtenidos anteriores. Se considera que habría una influencia de la aplicación del N químico y del estiércol, teniendo en cuenta buena condición ambiental para la absorción de este elemento. 


\section{CONCLUSIONES}

Para el rendimiento seco de la Brachiaria humidicola, fue el más alto en el T3, se considera que tuvo alta influencia de la aplicación nitrogenada adecuada. Por otra parte, también se observó la influencia por la aplicación del estiércol en los T4 y T5.Para el T5 se considera la aplicación nitrogenada elevada para el pasto gramíneo, teniendo en cuenta la cantidad aplicada del $\mathrm{N}$ químico más $1.99 \%$ del $\mathrm{N}$ como el valor contenido del estiércol con descomponerlo con el tiempo.

Por otra parte para las leguminosas nativas totales, se observó alto rendimiento seco en los $\mathrm{T} 2$ y $\mathrm{T} 4$, los valores fueron de 1139.6 y $976.0 \mathrm{~kg} / \mathrm{ha}$, respectivamente, mientras que para el T3 $443.9 \mathrm{~kg} / \mathrm{ha}$.

Para las leguminosas, se considera que el crecimiento fue vigoroso sin la aplicación nitrogenada química, teniendo en cuenta el capaz de la fijación biológica del $\mathrm{N}$ en el aire por Rhizobio puesto a la raíz de las plantas.

Relativamente, la proporción de la Brachiaria humidicola fue el más alta proporción de otras plantas en todos los tratamientos, especialmente la proporción de la Brachiaria humidicola en el T3 fue el más alto de la del mismo pasto gramíneo en otros tratamientos porque hubo alta influencia de la aplicación nitrogenada química.

Por otra parte, para las plantas leguminosas totales, se las observó alta proporción en los T2 y T4 porque no se aplicó el abono nitrogenado químico. Por otra parte, se observó baja proporción de las leguminosas totales en el T1 en comparación con las en los T2 y T4. Se considera que la actividad del Rhyzobio se inhibió en la condición de la deficiencia del $\mathrm{P}$ en el suelo.

En cada tratamiento, se observó distinto resultado, pero en los T2 y T4 la dominación del Desmodium barbatum fue alta, y Se considera que para el pasto leguminoso hubo mala influencia de la aplicación nitrogenada química y el nitrógeno con el estiércol, teniendo en cuenta poca descomposición con el N inorgánico liberado. Por el contrario, para el Calopogonium mucunoides la alta dominación en los T3 y T5, y se considera que prefirió el $\mathrm{N}$ aplicado con el estiércol a la condición sin la aplicación.

Suma por la cantidad absorbida del $\mathrm{N}$ en el T3 fue el más alta de la suma de otros tratamientos, y se considera que hubo influencia del gran crecimiento y la absorción para la Brachiaria humidicola. Pero para el Desmodium barbatum, la cantidad en el T3 fue más baja que la cantidad en los T2 y T4 porque era la condición sin la aplicación nitrogenada química. Totalmente, del punto de la vista de la suma por la cantidad del $\mathrm{N}$ (Material proteico), se pareció que los T2, T4 y T5 fueron altas adecuadas para el suministro a ganados criados, y los tratamientos tuvieron una balanza del pasto gramíneo y leguminoso en comparación con el T3.

A diferencia de los resultados del $\mathrm{N}$, la Suma por la cantidad absorbida en el T4 y el T5 fue el más alta de la suma de otros tratamientos, y se considera que hubo influencia de quelato producido al camino de la descomposición del estiércol aplicado, previno la fijación o la adsorción del $\mathrm{P}$ al coloide del suelo. Especialmente, para el Desmodium barbatum en el T2 fue alta absorción al igual que el caso en el T4. Se considera que el poder absorbido fosfatado sería fuerte en la condición sin la aplicación nitrogenada química.

Para la cantidad absorbida del $\mathrm{K}$, se observó casi misma tendencia del caso de la del N.

Para la suma de la cantidad absorbida del Ca, los valores en los T2, T3, T4 y T5 fueron similares, pero, para el T3, la balanza de la dominación de los pastos fue mala. 
A diferencia del resultado del caso del Ca, la suma del Mg en el T3 fue el más alta al igual que el caso de la suma del $\mathrm{N}$ y $\mathrm{K}$, pero la balanza de cada pasto en el T3 fue mala, por otra parte para el T2 y T4 fue la buena.

\section{REFERENCIAS}

ANAM (Autoridad Nacional del Ambiente, 2004). Programa Nacional de Lucha Contra la Desertificación

Ávila, M y Pasto, U. (1989). Brachiaria humidicola CIAT 679 (Rendle), Una alternativa para los suelos Baja Fertilidad y Áreas de prolongada sequía. Plegable IDIAP. pp 6
Díaz-Romeu, R., y Hunter, A. (1978). Metodologías de muestreo de suelos, análisis químico de suelos y tejido vegetal $\mathrm{y}$ de investigaciones en invernadero

Jaramillo, S. E. (1991). Pedones y campo y estaciones experimentales del IDIAP. Boletín Técnico No 38. Divisa, Panamá. pp. 67

Heckadon Moreno, S. (1984). La colonización campesina de bosques tropicales en Panamá. Colonización y Destrucción de Bosques en Panama. Panama: Asociación Panameña de Antropología, 17-44 